\title{
Control de la superficie y el volumen en la nanoescala para la configuración y el diseño de nanodispositivos
}

\author{
Edgar E González-Jiménez \\ Instituto Geofísico, Facultad de Ingeniería, Pontificia Universidad Javeriana, Bogotá, Colombia \\ Centro de Ciencia y Tecnología Nanoescalar, Bogotá, Colombia \\ Artículo de posesión para el ingreso como miembro correspondiente a la \\ Academia Colombiana de Ciencias Exactas, Físicas y Naturales el 10 de agosto de 2016
}

\begin{abstract}
Resumen
Con el uso de nanopartículas coloidales con volumen interno y superficie de interacción mejorada, se investigaron las condiciones geométricas requeridas para optimizar la sensibilidad de la resonancia del plasmón superficial en función de los cambios en la superficie por adsorción de entidades atómicas o moleculares. Debido a sus propiedades es posible configurar estas nanopartículas para el diseño de nanosensores y nanoantenas en modo de absorción o emisión. Se verificó que en el volumen encerrado se incrementó la intensidad del campo eléctrico, conformándose las llamadas "cavidades calientes", de gran utilidad en el campo de la nanomedicina para mejorar el transporte y la entrega controlada de medicamentos. (C) 2016. Acad. Colomb. Cienc. Ex. Fis. Nat.
\end{abstract}

Palabras clave: Nanopartículas huecas; Nanosensores; Nanoantenas; Nanorreactores; Plasmón superficial; Oxidación galvánica; Efecto Kirkendall.

Surface and volume control at the nanoscale for nanodevices configuration and design

\begin{abstract}
The study of the geometric conditions required to optimize the sensitivity of the surface plasmon resonance as a function of the adsorption of atomic or molecular entities is possible using colloidal nanoparticles with internal volume and improved interaction surface. Given its properties, it is possible to configure these nanoparticles for the design of nanosensors and nanoantennas in absorption or emission mode. In the enclosed volume, the electric field intensity is increased forming "hot cavities", which are useful in nanomedicine for controlled drug delivery. (C) 2016. Acad. Colomb. Cienc. Ex. Fis. Nat.
\end{abstract}

Key words: Hollow nanoparticles; Nanosensors; Nanoreactors; Nanoantennas; Surface plasmon; Galvanic oxidation; Kirkendall effect.

\section{Introducción}

Una de las principales diferencias que caracterizan la nueva generación de nanomateriales, específicamente las nanopartículas coloidales, es el incremento de la relación entre superficie y volumen a medida que el tamaño de la nanopartícula se reduce. En las nanopartículas sólidas de unos pocos nanómetros de diámetro, el número de átomos que ocupan la superficie exterior es mayor que el número de átomos en el volumen encerrado. Esta superioridad de superficie favorece el incremento de la energía interfacial, la reactividad de la nanopartícula, la eficiencia en la absorción y la capacidad de hacerla funcional de forma eficiente con entidades moleculares de interés (Sperling \& Parak, 2011).

En el camino hacia un mayor control de la complejidad en la forma y la composición de las nanopartículas coloidales, manteniendo la monodispersidad en el tamaño (Parak, 2011; Ibáñez \& Cabot, 2013), se han conseguido desarrollos sintéticos que permiten, además de la superficie externa, producir superficies en el interior de la partícula $(\mathbf{O h}, \boldsymbol{e t} \boldsymbol{a l}$., 2013; Yin, et al., 2004). Este tipo de materiales, que pueden clasificarse como nanopartículas coloidales "huecas", incrementan sustancialmente el valor del área de interacción con respecto a la contraparte sólida si están configuradas para tener acceso al interior, lo cual favorece el valor de utilidad y el rango de uso en la catálisis, los nanosensores, la fijación e inmovilización de agentes químicos en procesos de recuperación ambiental, el transporte y la entrega controlada de medicamentos, y las reacciones químicas encapsuladas, entre muchas otras aplicaciones (Wang, et al., 2009; González, et al., 2016).

\section{Correspondencia:}

Edgar E. González, egonzale@javeriana.edu.co

Recibido: 19 de julio de 2016

Aceptado: 3 de noviembre de 2016 
Aunque la transición hacia el desarrollo de capacidades para la manipulación y la programación de la superficie en materiales, más allá de la tecnología bulk, ha sido una de las más destacadas contribuciones a la revolución nanotecnológica emergente, la generación de nanomateriales con dominios volumétricos internos como las nanopartículas coloidales huecas, está abriéndole el camino a una nueva alternativa para capitalizar las ventajas que ofrece la escala nanométrica. Esta generación permite ampliar la librería de nanomateriales disponibles que incorporan nuevas formas geométricas y de distribución y disposición de los componentes atómicos (González, et al., 2011).

Las nanopartículas coloidales huecas dotadas con poros de acceso pueden considerarse "laboratorios nanoescalares" que hacen uso integral de la superficie y el volumen para la realización de complejas tareas biológicas, físicas y químicas de elevada precisión y localización espacio-temporal. En ellas es posible configurar espacios capaces de confinar y controlar reacciones químicas, los nanorreactores, (Subramanian, et al., 2016), y generar condiciones que incrementan la intensidad de la radiación electromagnética incidente para mediciones analíticas (Liu, et al., 2016), así como el diseño de nanoantenas (Denkova, et al., 2013) y sofisticados sensores para la detección y la medición en escala molecular (Mahmoud, et al., 2014).

Las nanopartículas metálicas, específicamente aquellas conformadas por metales nobles como el oro y la plata, presentan algunas ventajas significativas en el comportamiento óptico y térmico con respecto a las nanopartículas de otros tipos de composición. Además, exhiben respuesta en el espectro visible debido a sus reducidos tiempos de relajación electrónica y elevadas frecuencias de plasmón y de transición entre bandas. Además de la posibilidad de diseñar y manufacturar arquitecturas que privilegien holísticamente el área superficial y el volumen en escala nanométrica, estas ventajas permiten introducir una nueva variedad de estructuras en el contexto de los nanomateriales que favorecen el tránsito hacia la ciencia e ingeniería de las nanocavidades, las cuales harán viables los usos mencionados.

En este trabajo se determinó la disponibilidad del volumen, y la posibilidad de optimizar la superficie en nanopartículas coloidales metálicas sintetizadas mediante el control de la morfología, la composición y el tamaño, lo cual permite la formación de cavidades y poros. Se evaluó, asimismo, la utilización de estos materiales para mejorar los mecanismos involucrados en las plataformas de sensado, el transporte y la entrega controlada de medicamentos, así como la configuración de nanorreactores y nanoantenas.

\section{Materiales y métodos}

Para la síntesis de las nanopartículas metálicas se utilizaron las siguientes sustancias químicas: etilenglicol anhidro al $99,8 \%$, ácido ascórbico al $99 \%$, nitrato de plata a $>99,9 \%$, tetracloropaladato (II) de sodio, clorhidrato de oro al 99,99\%, polivinilpirrolidona (PVP) con peso molecular de $55 \mathrm{~K}$, bromuro de hexadeciltrimetilamonio, sulfuro de sodio nonahidratado al 99,9\%, hexacloroplatinato (IV) de potasio, cloruro de sodio, l-cisteína, y poli( $\mathrm{N}$ isopropilacrilamida). Todos los compuestos químicos se adquirieron de Sigma Aldrich.

En la síntesis de nanopartículas polimetálicas huecas y porosas con control en la forma, tamaño y composición, se utilizó el método reportado en González, et al. (2011), el cual recurre al reemplazo galvánico y al efecto Kirkendall para "tallar" las correspondientes estructuras huecas y porosas a partir de partículas que sirven de "plantillas de sacrificio".

En un típico protocolo de síntesis, se parte de una nanopartícula metálica que juega el papel de soporte sobre el cual se produce el proceso galvánico de remoción de átomos por oxidación y el correspondiente reemplazo por otros de mayor potencial estándar de reducción. Así, se puede usar la plata para la plantilla de sacrificio y emplear una o dos sales metálicas como agentes oxidantes. Para nanopartículas polimetálicas con cavidades interiores, se inicia con un proceso de tipo oxidativo producido con una sal metálica, el cual genera un patrón de cavidades que puede ser controlado con el tiempo de exposición, el tipo de sal oxidativa utilizada y un surfactante (González, et al., 2011). Así, en el caso de plantillas cúbicas de plata en medio acuoso y en concentraciones de $10 \mathrm{~nm}$, utilizadas con el fin de formar cavidades internas poliédricas, se usa una sal metálica con un mayor potencial de reducción que la plata, por ejemplo, el tetracloropaladato (II) de sodio en concentraciones de $3,5 \times 10^{-3} \mathrm{M}$ y se agrega bromuro de hexadeciltrimetilamonio en concentraciones de $8,6 \times 10^{-3} \mathrm{M}$. Ello permite que el proceso de oxidación se inicie en ciertos sitios específicos, tales como las esquinas o el centro de las caras externas de las nanopartículas. Mediante el reemplazo galvánico en estos sitios específicos, se forman cavidades internas aún incompletas, pero cuyo tamaño puede ser controlado con el tiempo y la relación molar entre la sal de paladio y la plata. Todo el proceso de síntesis se hace en solución acuosa y a temperatura ambiente.

Para afinar la forma y el tamaño de las cavidades, así como la formación de espacios entre dichas cavidades, se hace uso de una segunda sal con un potencial de reducción mayor al anterior (por ejemplo, clorhidrato de oro). En estas condiciones, y en presencia del bromuro de hexadeciltrimetilamonio, el cual aporta átomos de bromo al cristal de plata según se ha podido corroborar (González, et al., 2011), es posible reducir el coeficiente de difusión y, en consecuencia, favorecer el efecto Kirkendall, el cual produce un tallado mucho más fino que aquel que se produce por reemplazo galvánico. Cuando se requieren nanopartículas con una sola cavidad, se emplea únicamente la sal de oro como agente oxidante.

Para la síntesis de las nanopartículas de plata que sirven de soporte para la formación de las nanopartículas metálicas porosas, se usa el método poliol (Fievet, et al., 1989; Skrabalak, et al., 2008). En este proceso de síntesis 
se hace uso de un poliol (por ejemplo, Etilenglicol), que a temperaturas de $160^{\circ} \mathrm{C}$ y con un potencial de oxidación de $1,65 \mathrm{eV}$, reduce las sales metálicas, en particular el nitrato de plata. Como agente estabilizante se utiliza PVP, la cual posee una elevada constante dieléctrica y se disuelve fácilmente con el etilenglicol. Con relaciones molares $\mathrm{PVP} / \mathrm{AgNO}_{3} \approx 1,5, \mathrm{y}$ concentraciones de nitrato de plata en el rango de 0,125 a $0,25 \mathrm{M}$, se obtienen nanopartículas cúbicas. Si se mantiene la misma relación molar entre la PVP y el nitrato de plata, pero se reduce la concentración de la sal de plata por debajo de $0,1 \mathrm{M}$, es posible obtener nanopartículas de geometría cilíndrica, los llamados nanoalambres. Con una relación molar $\mathrm{PVP} / \mathrm{AgNO}_{3}>5$, se obtienen nanopartículas esféricas.

Para el estudio de cambios en el plasmón superficial cuando las nanopartículas huecas permiten la interacción únicamente con la superficie externa y con la superficie total (interna y externa), previamente recubiertas con pNIPAAm, estas se funcionalizan con 1-cisteína disuelta en agua mediante inmersión durante cuatro horas a temperatura ambiente en una concentración de $10 \mathrm{mM}$.
Para la caracterización de las nanopartículas, tanto las utilizadas como plantilla de sacrificio como las resultantes de los procesos de sustitución galvánica y de efecto difusivo del tipo Kirkendall, se usó un microscopio electrónico de transmisión Jeol de 120 kV, y un espectrofotómetro Shimadzu UV-Visible UV-2600 para un sistema de difracción de rayos-X X'Pert Pro MPD.

\section{Resultados}

Con base en los registros fotográficos con microscopía electrónica de transmisión de las muestras de nanocubos de plata (figura $1 \mathrm{a}, \mathrm{b}$ ), se obtuvo un tamaño medio de arista $45 \pm 4$ nm. A partir de los resultados obtenidos se verificó que los nanocubos de plata correspondieron a monocristales (Figura 1 c), los cuales permiten obtener nanopartículas huecas de alta calidad cristalina y de forma y tamaño similares mediante reemplazo galvánico y crecimiento epitaxial. De otra parte, el espectro de absorbancia para la muestra de nanocubos sintetizados mostró dos picos de resonancia: el primero se localizó en $352 \mathrm{~nm}$ y correspondió al modo de resonancia dipolar característico de las estructuras cúbicas a)

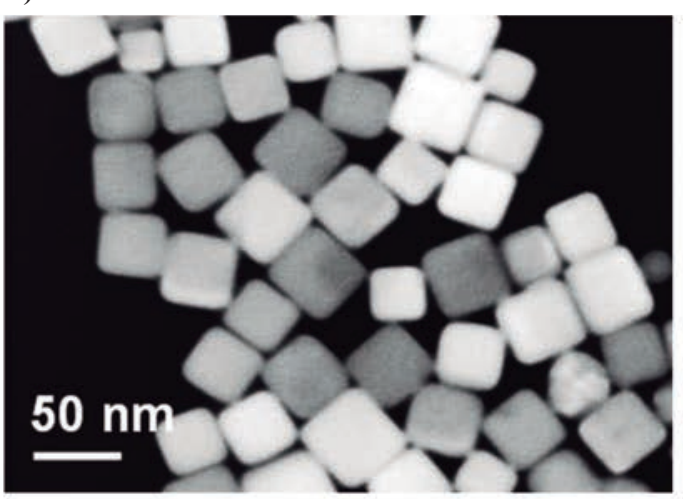

b)



c)

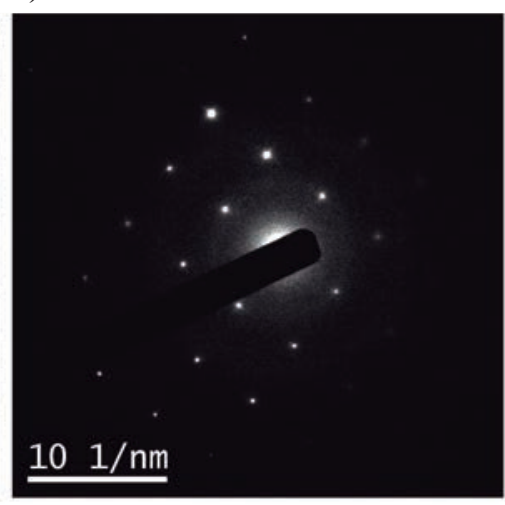

d)

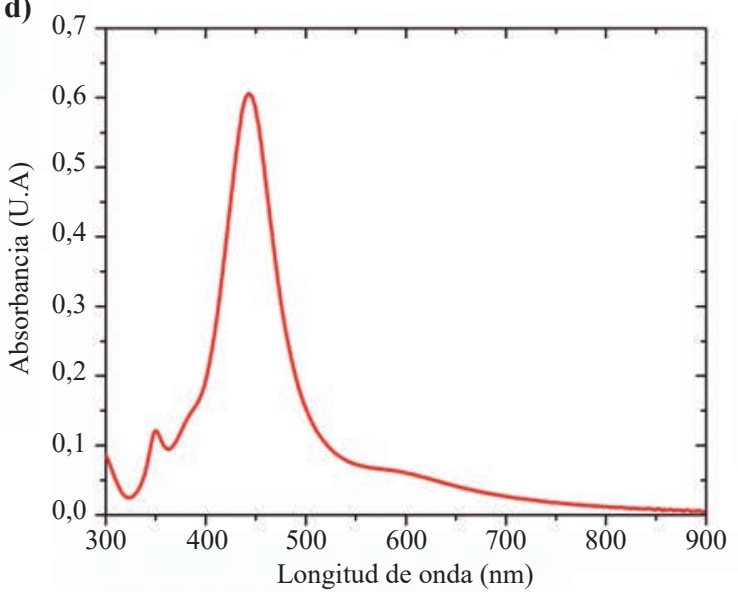

e)

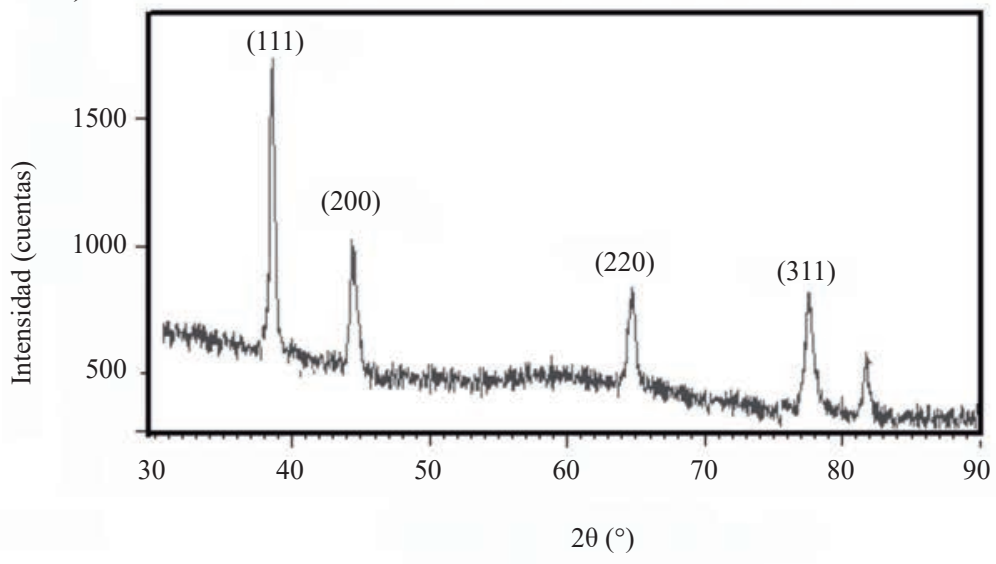

Figura 1. Caracterización de los nanocubos de plata. a) Imagen de microscopio electrónico de transmisión de una muestra de nanocubos de plata utilizados para la síntesis de las nanopartículas huecas. b) Imagen detallada de un nanocubo de plata con longitud de arista L. c) Patrón de difracción de electrones de área selecta (selected area electron difraction, SAED) de los nanocubos de plata. d) Espectro Uv-Vis de nanocubos de plata. e) Patrón de difracción de rayos X de los nanocubos de plata 
debido a los vértices, y el segundo pico se asoció a una longitud de onda de $449 \mathrm{~nm}$ correspondiente al modo de resonancia cuadrupolar (figura 1d).

Las nanopartículas cúbicas de plata presentaron un patrón de difracción de rayos X con picos de (111), (200), (220), (311), tal como se muestra en la figura 1e, los cuales pueden indexarse en una estructura cúbica centrada en las caras (fcc).

La figura 2 a corresponde a una imagen tomada con microscopía electrónica de transmisión en una muestra de nanopartículas huecas obtenidas a partir de la galvanización ejercida sobre los nanocubos de plata, los cuales exhibían una capa de oro en la superficie exterior y la aleación de plata $\mathrm{y}$ oro en el interior de la pared como resultado del método utilizado en la síntesis (González, et al., 2011). Como puede observarse en la figura $2 \mathrm{~b}$, el patrón de difracción de los rayos-X corroboró que, al igual que en los nanocubos de plata, se trata de estructuras monocristalinas del tipo $f_{c c}$ con una elevada calidad estructural.

Las nanopartículas huecas de una pared presentaron un pico de resonancia para una longitud de onda de 640 $\mathrm{nm}$, tal como se aprecia en el espectro de absorbancia de la figura 3a, con un ancho de banda cercano a los $200 \mathrm{~nm}$. Un aspecto de gran importancia para la aplicación en tareas de sensado en este tipo de estructuras es la sensibilidad de la posición del pico de resonancia en función del ambiente que rodea la partícula, en particular en la región interfacial por adsorción de agentes moleculares. Para evaluar la eficiencia de la nanopartícula como sensor en términos de capacidad para entregar valores de calidad, se calcula la figura de mérito, definida como la relación entre el índice de refracción y el ancho de banda de la región de resonancia. Esta cantidad depende drásticamente del ancho de la pared $\mathrm{D}$ de la partícula, tal como se muestra en la gráfica de la figura 3b. Esta curva se obtuvo numéricamente a partir del procedimiento reportado en Cao, et al. (2009).

El ancho de la pared se puede controlar con el tiempo de reacción oxidativa en el proceso de síntesis y la concentración de la sal precursora. En el caso de las partículas huecas obtenidas, se logró que el ancho de la pared se aproximara a 5,5 nm, valor situado en el intervalo en el cual se produce un máximo valor de la figura de mérito, tal como puede apreciarse en la gráfica de la figura 3b. En consecuencia, estas nanopartículas huecas de pared simple, con las condiciones a)

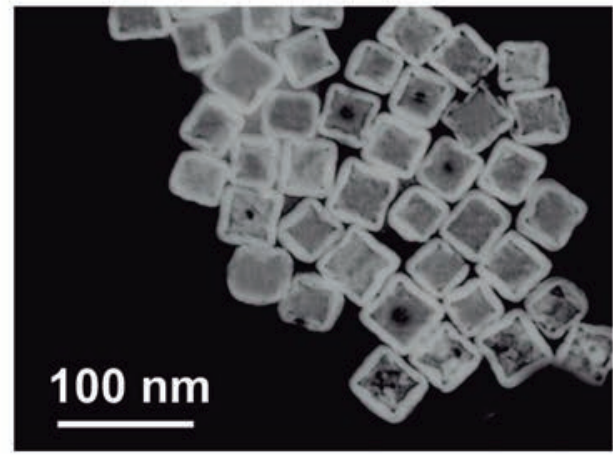

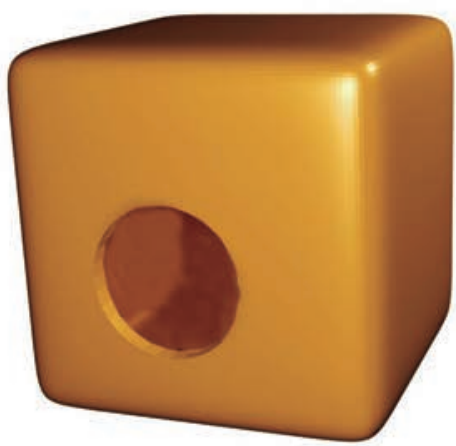

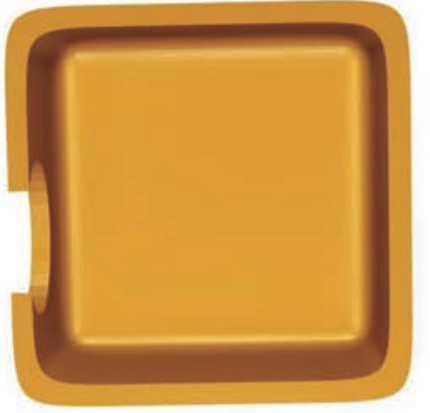

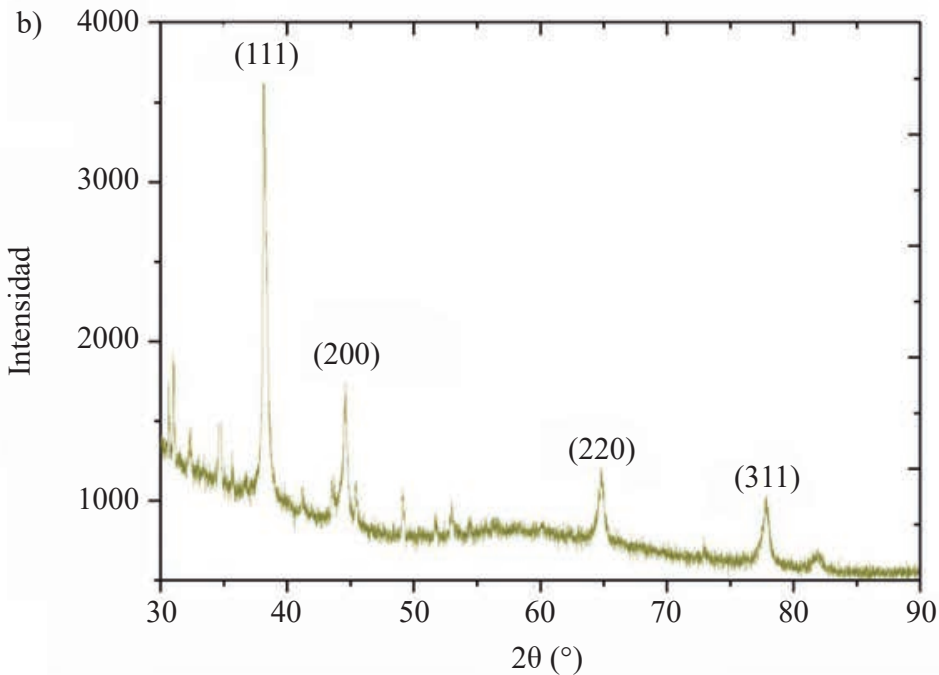

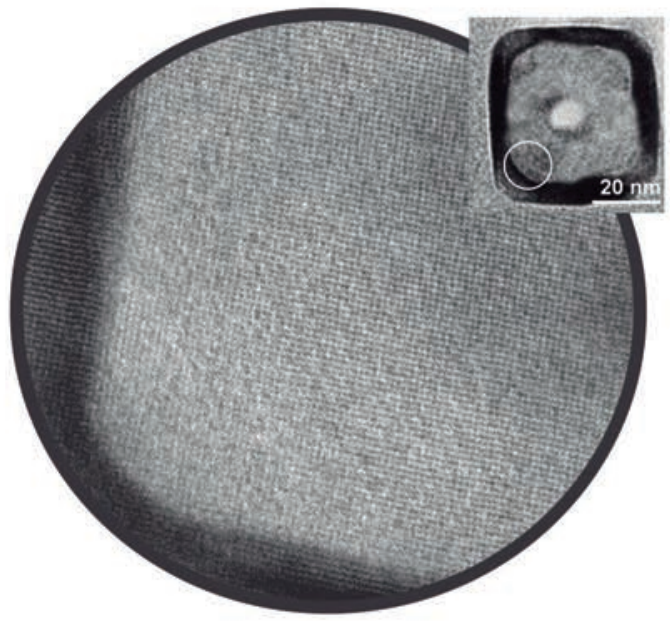

Figura 2. a) Imagen obtenida con microscopía electrónica de transmisión de nanopartículas huecas sintetizadas a partir de plantillas de geometría cúbica. Se ilustra un diagrama tridimensional y de corte transversal. b) Patrón de difracción de rayos X y fotografía en alta resolución de uno de los vértices de la partícula. 

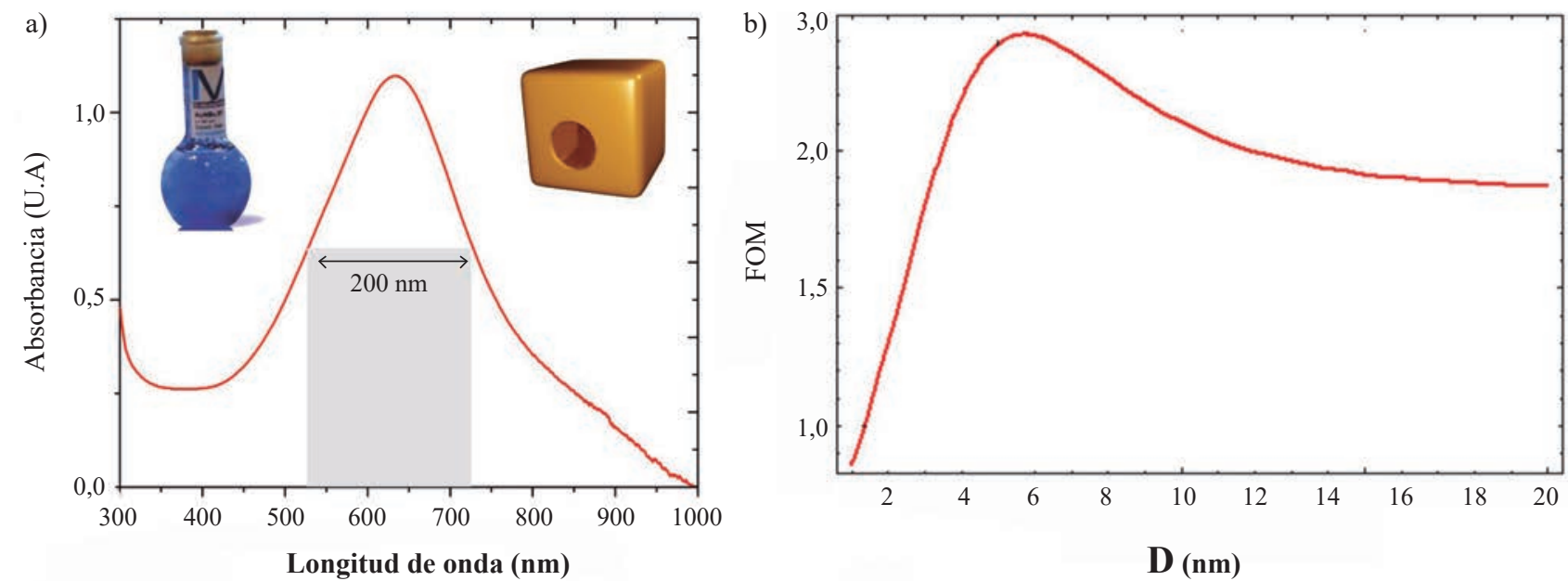

c)

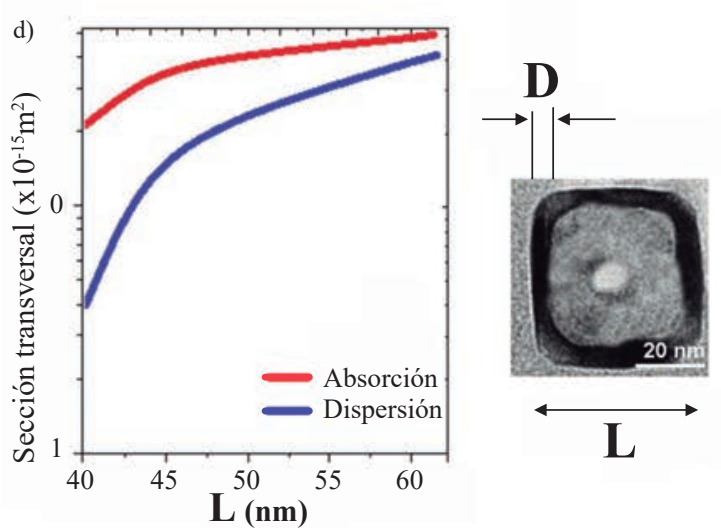

Figura 3. Espectro de absorbancia para nanopartículas huecas de pared simple. b) Figura de mérito de las nanopartículas de una pared en función del espesor de la pared. c) Ventana de agua para la absorción en función de la longitud de onda. d) Absorción y emisión en función de la longitud de arista de las nanopartículas de pared simple.

geométricas y valores de longitud obtenidos, presentaban una condición óptima para su uso como nanosensores de resonancia del plasmón superficial.

Un aspecto fundamental para el uso de estas nanopartículas en el transporte y entrega controlada de medicamentos, es la posibilidad de ser excitadas térmicamente con luz coherente perteneciente a la primera ventana de agua. En esta ventana, correspondiente al intervalo de longitudes de onda de 650 a $900 \mathrm{~nm}$ (figura 3c), los tejidos y el flujo sanguíneo resultan transparentes a la radiación. Las nanopartículas huecas sintetizadas presentaron un ancho de banda de resonancia en el intervalo de 530 a $750 \mathrm{~nm}$, el cual se interseca con la primera ventana de agua.

Al modificar la longitud de la arista externa L, manteniendo constante el ancho D de la pared de las nanopartículas, es posible modular la capacidad de absorción o dispersión de la radiación óptica. Así, para longitudes cercanas a los 60 $\mathrm{nm}$, la absorción se hace menos dominante con respecto a la dispersión. En estas longitudes, las nanopartículas pueden utilizarse como nanoantenas emisoras. En longitudes del orden de los $40 \mathrm{~nm}$, las nanopartículas se comportan como nanoantenas térmicas, ya que la absorción se hace dominante sobre la dispersión. Este comportamiento se ilustra en la gráfica de la figura $3 \mathrm{~d}$, obtenida numéricamente a partir del método reportado en Cao, et al. (2009).

El valor de área superficial efectiva de cada una de las estructuras sintetizadas se obtuvo a partir de los valores promedios de las longitudes de aristas y valores de longitud interna medidos de los registros de microscopía electrónica de transmisión. En el caso de las cajas de una sola pared (Figura 4), la longitud promedio de la arista de la superficie externa, $\mathrm{L}_{\mathrm{e}}$, resultó igual a $49 \pm 4 \mathrm{~nm}$, de la cual se obtuvo un valor teórico de área total superficial externa igual a $14.406 \mathrm{~nm}^{2}$. De otra parte, el valor de longitud promedio de la cavidad, $\mathrm{L}_{\mathrm{i}}$, fue de $38 \pm 5 \mathrm{~nm}$, lo cual generó un valor del área de la superficie interior igual a $8.664 \mathrm{~nm}^{2}$. El área total superficial teóricamente utilizable para la funcionalización y otros usos de interés de estas nanopartículas fue de $2,3 \times 10^{4} \mathrm{~nm}^{2}$. El valor de área superficial disponible para inmovilizar agentes de interés, tales como moléculas, iones, etc., determina drásticamente la capacidad de interacción y adsorción que poseen esta nanoestructuras, lo cual resulta estratégico no solamente para el sensado por el plasmón superficial sino, además, para su uso en procesos químicos confinados o nanorreactores. 

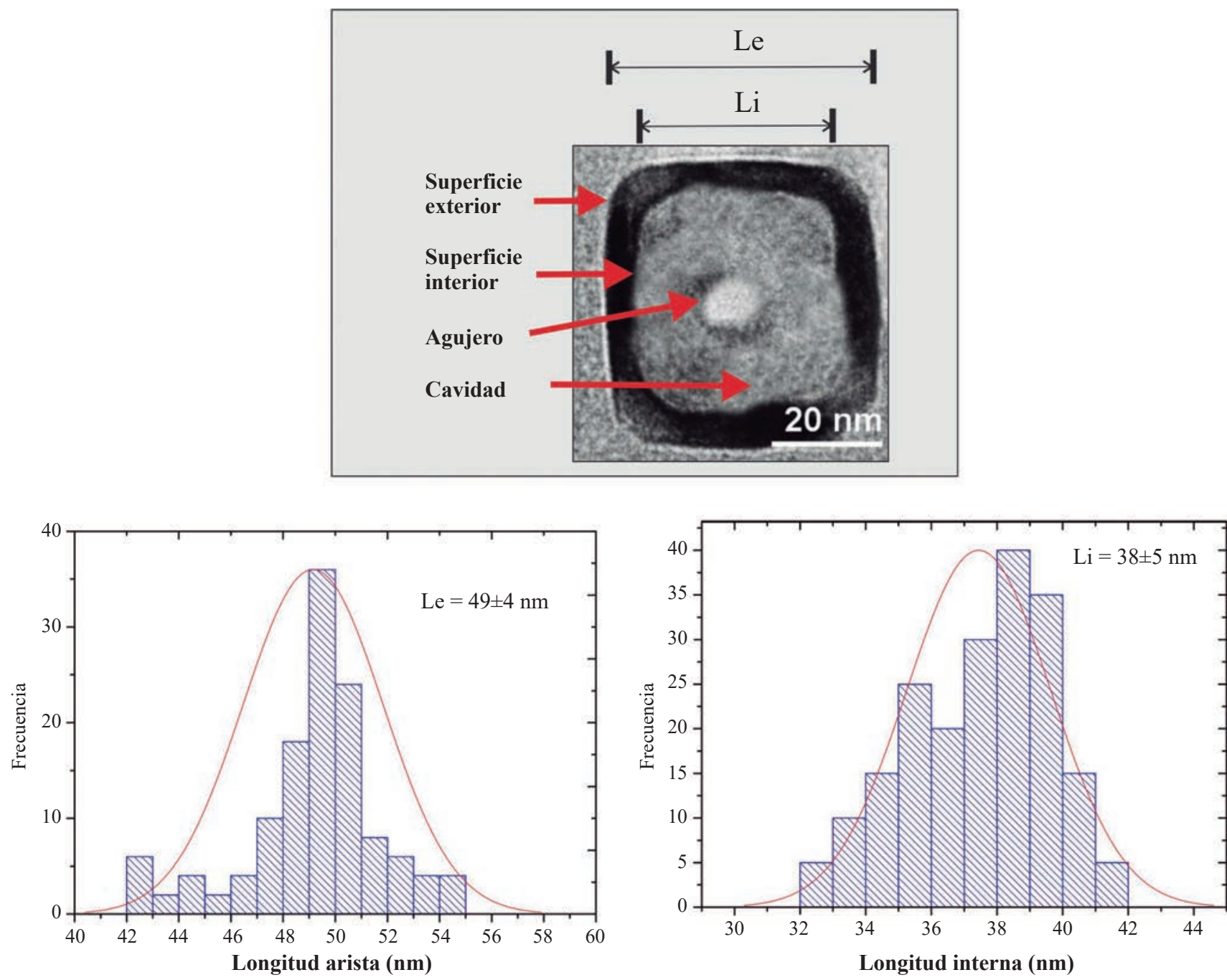

Figura 4. Valores promedio de la arista externa, $\mathrm{L}_{\mathrm{e}}$, y longitud interna, $\mathrm{L}_{\mathrm{i}}$, de las nanopartículas coloidales huecas de pared simple obtenidos de mediciones de registros de microscopía electrónica de transmisión. Estos valores se requieren para calcular el área superficial efectiva.

Para evidenciar el papel que juega el volumen y, en consecuencia, el incremento de superficie, se funcionalizó superficialmente una muestra de nanopartículas en solución acuosa con 1-cisteína, la cual posee un grupo tiol que la hace afín con la superficie de oro de la nanopartícula. Para cerrar o abrir el agujero de la nanopartícula que permite el acceso al interior, se utilizó un polímero termosensible como el poli(N-isopropilacrilamida) (pNIPAAm), el cual exhibe una temperatura crítica de $32{ }^{\circ} \mathrm{C}$, por debajo de la cual su comportamiento resulta hidrofílico, mientras que con temperaturas mayores a $32{ }^{\circ} \mathrm{C}$ se hace hidrofóbico. Esto permitió controlar mediante la temperatura la transición que abre o cierra el agujero de las partículas huecas. El polímero pNIPAAm es hidrofílico a temperatura ambiente, con la cual se mantiene cerrado el poro, mientras que a $35^{\circ} \mathrm{C}$ el polímero transita a un comportamiento hidrofóbico que permite la apertura del poro y, en consecuencia, la interacción de la 1-cisteína con la superficie interna de las nanopartículas. La figura 5 muestra los espectros de absorbancia obtenidos para una muestra coloidal de nanopartículas cubiertas únicamente con pNIPAAm de una muestra funcionalizada con 1-cisteína a temperatura ambiente y, posteriormente, con 1-cisteína a una temperatura de $35{ }^{\circ} \mathrm{C}$. Se observa en esta un corrimiento perceptible del pico de resonancia con el cambio del área superficial disponible para la fijación de la l-cisteína, la cual se incrementó cuando el poro se encontraba abierto.

Las cavidades en nanopartículas coloidales ofrecen una interesante posibilidad de utilizarlas como nanohornos para la activación de reacciones químicas en su interior y posterior entrega de productos al exterior a través del poro presente en cada nanopartícula. Para evaluar la interacción del campo eléctromagnético con las nanopartículas huecas provistas de un poro de acceso, se empleó el método de Yee (Yu, et al., 2009), en el cual se resuelven las ecuaciones de Maxwell a partir de la discretización del espacio y del tiempo. Para esta simulación se escogió una partícula de arista de 49 $\mathrm{nm}$, con un espesor de pared de $7 \mathrm{~nm}$ y un diámetro del agujero de acceso al interior de $13 \mathrm{~nm}$. La figura 6 muestra 
el resultado obtenido para la componente de campo eléctrico incidente polarizado en dirección paralela a la pared provista de agujero. La frecuencia de la onda electromagnética correspondiente a la frecuencia del pico de resonancia del plasmón de las nanopartículas fue de $5 \times 10^{5} \mathrm{GHz}$ según el espectro de resonancia. Se observó un incremento en la intensidad del campo eléctrico en la región del agujero, así como en el interior de la cavidad (Figura 6). Estas regiones pueden considerarse como "regiones calientes".

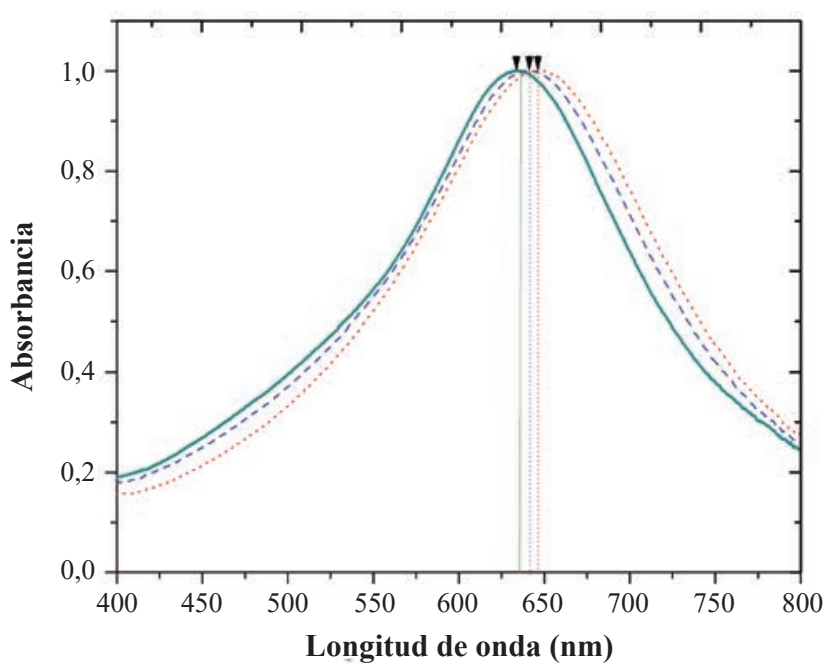

Figura 5. La línea continua corresponde al espectro de absorbancia para una muestra coloidal de nanopartículas funcionalizadas con pNIPAAm. La línea a trazos corresponde a la muestra funcionalizada con cisteína con el poro cerrado en cada partícula. La línea punteada corresponde a partículas que tienen el poro abierto con acceso al interior.
Aziz, et al. (2016) reportaron un estudio de resonancia del plasmón en nanopartículas huecas de una sola pared sintonizado en el rango del ultravioleta al infrarrojo cercano. Los autores mapearon las resonancias del plamón con espectroscopía de pérdida de energía de electrones, y observaron una distribución homogénea de altos valores de resonancia en torno a las nanopartículas huecas.

Para ilustrar el incremento del área superficial de interacción en función de la morfología interna de la partícula, se sintetizaron nanopartículas con dos cavidades internas, con una capa de oro externa y una aleación de plata y paladio en el interior de las paredes, lo cual se verificó con espectroscopía de pérdida de energía de electrones. Como se ilustra en la imagen tomada con microscopía electrónica de transmisión de la Figura 7a, la cavidad menor interna presentó una disposición arbitraria dentro de la cavidad principal de la nanopartícula. La longitud de la arista resultó similar a las de las nanopartículas de una sola pared, ya que para esta síntesis se utilizaron los mismos nanocubos de plata como plantillas de sacrificio. Se muestra, además, una gráfica tridimensional de la correspondiente nanopartícula con un corte que permite apreciar el interior, el corte transversal y el espectro de absorción. Como se puede apreciar, el pico de resonancia del plasmón superficial no se modificó significativamente con respecto al obtenido para las nanopartículas de una sola pared (figura 7b). El cálculo del campo eléctrico incidente, con frecuencia de $5 \times 10^{5} \mathrm{GHz}$, de las nanopartículas con dos cavidades se presenta en la figura 7c. Fue visible un incremento del campo dentro de las dos cavidades, las cuales se comportaron como "nanocuernos" conectados, útiles para aplicaciones que requieren reacciones químicas controladas, selectivas y encapsuladas.
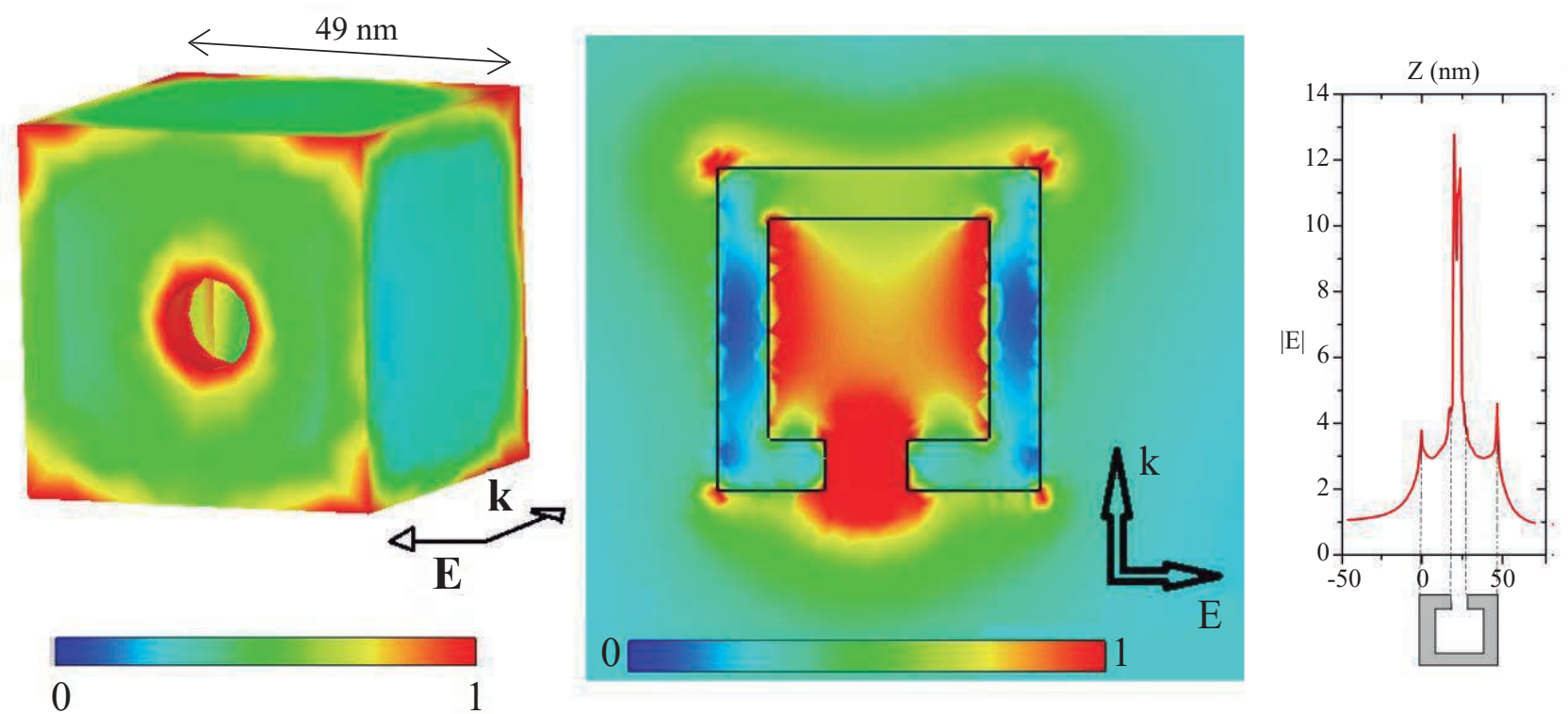

Figura 6. Campo eléctrico en torno a una nanopartícula hueca de pared simple con un poro de $13 \mathrm{~nm}$ de diámetro y 49 nm de arista. El vector de onda de la radiación polarizada incidente está dirigido en dirección perpendicular a la pared que se encuentra agujereada. Se muestra un corte transversal y la magnitud del campo eléctrico en la superficie que contiene el agujero. 
a)



b)

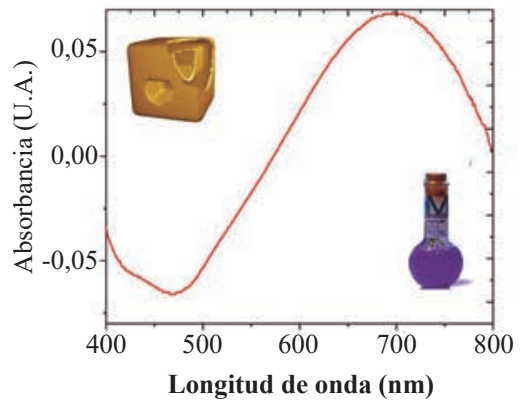

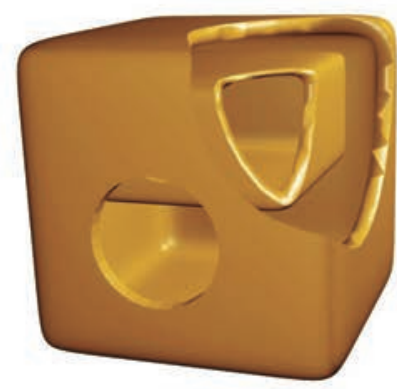

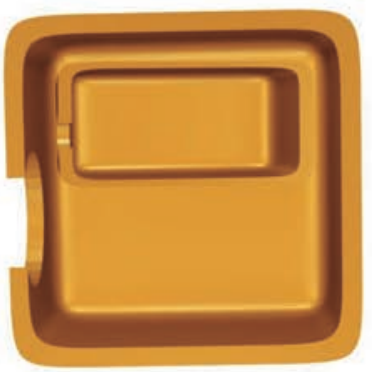

c)

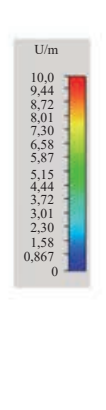

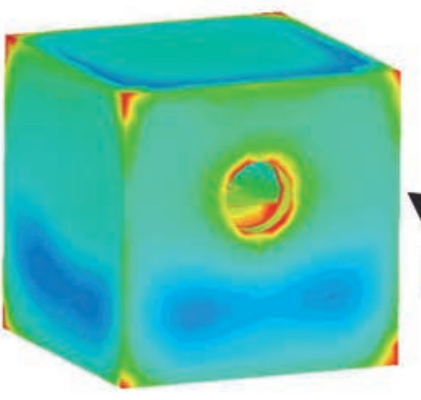



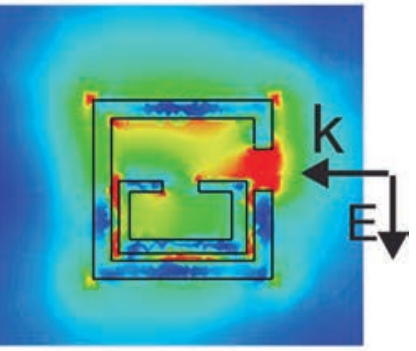

Figura 7. a) Imagen tomada con microscopía electrónica de transmisión de nanopartículas huecas con dos cavidades poliédricas. Se ilustra una imagen tridimensional y corte. b) Espectro de absorbancia. c) Distribución de la intensidad del campo eléctrico incidente

A partir de los valores medios de las longitudes de las aristas y las cavidades fue posible determinar la superficie efectiva de interacción, la cual corresponde a $3,1 \times 10^{4} \mathrm{~nm}^{2}$. Al comparar este resultado con el de las partículas de una sola pared y las partículas sólidas, se obtiene la gráfica comparativa de la figura 8 , que indica claramente un incremento apreciable a medida que aparecen las particiones del volumen interno de las nanopartículas. Cabe anotar que mediante microscopía electrónica se verificó que las cavidades de las nanopartículas presentaban un poro de acceso.

\section{Discusión}

En este estudio se sintetizaron estructuras huecas de pared simple con acceso al interior a través de un poro en la superficie, lo cual permite el acceso tanto al volumen como a la superficie interior. La presencia de este poro es una de las principales diferencias y ventajas que ofrece el método empleado para su síntesis. Durante la formación de la nanopartícula, el agujero inicial en donde se inicia la oxidación de la plantilla de sacrificio (corrosión por picadura) se mantiene abierto hasta finalizar el proceso galvánico. Esto no ocurre con otras rutas de síntesis de partículas metálicas huecas, en las que el agujero inicial se cierra por completo (Skrabalak, et al., 2008).

La presencia de "regiones calientes" en el agujero y en las cavidades resultó estratégica, ya que con muy poca energía de radiación incidente se logró la activación de polímeros termosensibles ubicados en la zona de acceso a la cavidad, así como incrementos de temperatura en su interior,



Figura 8. Valores comparativos del área superficial de interacción disponible para nanopartículas sólidas, huecas y de dos cavidades, respectivamente.

lo cual favorece el transporte difusivo hacia el exterior. De otra parte, el uso del volúmenes y superficies interiores, mejora los protocolos de liberación de medicamentos con nanopartículas metálicas, ya que las nanopartículas sólidas ofrecen exclusivamente la superficie exterior para disponer las moléculas de interés, lo cual dificulta una liberación controlada y una óptima cantidad de carga. Cuando la nanopartícula sólida alcanza el blanco, por ejemplo la superficie 
tumoral, se libera por completo la carga, lo cual impide la entrega en zonas tumorales más profundas. El hecho de contar con una superficie y un volumen interior incrementa notablemente la capacidad de carga de la molécula de interés, así como la posibilidad de hacer una entrega mucho más controlada en tiempo y lugar.

La posibilidad de controlar el espesor de la pared de las nanopartículas a partir de parámetros como la concentración del precursor y el tiempo de reacción, permite modular su respuesta y su sensibilidad a la radiación óptica incidente y ello da lugar a la transición del modo dominante de absorción, en el cual prevalece una respuesta resonante, a un modo dispersivo. Esta programación en la respuesta frente a la excitación por radiación abre la posibilidad de usar estas estructuras en el campo de la nanomedicina, específicamente en tratamientos en los que se requiere el modo de absorción, o para el diagnóstico, en el cual es más ventajoso el comportamiento dispersivo.

Actualmente nuestro equipo está investigando alternativas para mejorar el método de síntesis de precursores y solventes, así como de estabilizantes y reductores, para aproximarlo a la química verde. Ya se han obtenido interesantes resultados a temperatura ambiente y con el uso de extractos de frutos y hojas de plantas. De consolidarse, tales resultados permitirían la producción y la obtención de los componentes requeridos a gran escala y sin impacto ambiental, así como dispersiones de gran estabilidad.

\section{Conclusiones}

A partir de la síntesis de nanopartículas metálicas huecas con un poro de acceso al interior, se evaluaron experimental y teóricamente las condiciones para mejorar su uso y aplicación. La superficie efectiva de interacción de las nanopartículas de una sola pared se incrementó aproximadamente en un $40 \%$ con respecto a la contraparte sólida. Dado que las partículas presentaron un poro, la superficie $\mathrm{y}$ volumen interior quedaron habilitadas para incorporar entidades químicas o biológicas y transportarlas, o para lograr reacciones químicas encapsuladas.

Otra de las ventajas de estas nanopartículas cúbicas huecas de pared simple, es la posibilidad de modular su respuesta a la radiación óptica incidente en función de parámetros tales como el espesor de la pared y la longitud de la arista. Así, se verificó que la sensibilidad de las nanopartículas a los cambios en la interface producidos por la adsorción de átomos o moléculas en la superficie, dependió drásticamente del espesor de la pared, lo cual permitió establecer los valores de espesor de la pared para los cuales se maximiza la figura de mérito. Para nanopartículas de $49 \mathrm{~nm}$ de arista, se encontró que el valor del espesor en el cual se optimiza la capacidad de sensado por el plasmón superficial estaba entre los 4 y los 8 nanómetros.

No cabe duda de que la transición hacia la producción de nanomateriales con una mayor superficie de interacción, así como la opción de contar con el volumen en la escala nanométrica, abre una importante ruta de investigación para la búsqueda de nuevos y mejores métodos de síntesis que permiten obtener nanoestructuras con novedosas propiedades en un contexto de sostenibilidad y bajo impacto ambiental en seres vivos, y con un amplio espectro de aplicación.

\section{Conflicto de intereses}

El autor declara que no tiene conflicto de intereses.

\section{Agradecimientos}

Al Instituto Geofísico de la Facultad de Ingeniería de la Pontificia Universidad Javeriana y al Centro de Ciencia y Tecnología Nanoescalar, por su apoyo en la caracterización y el desarrollo computacional.

\section{Referencias}

Aziz, G., Patarroyo, J., Parramon, S., Arenal, Raul., Duchamp, M., González, E., Henrard, L., Bastús, N.G., DuninBorkowski, R.E., Puntes, V.F., Arbiol, J. (2016). Tuning the plasmonic response up: Hollow cuboid metal nanostructures. ACS Photonics. 3 (5): 770-779.

Cao, M., Wang, M., Gu, N. (2009). Optimized surface plasmon resonance sensitivity of gold nanoboxes for sensing applications. J. Phys. Chem. C. 113 (4): 1217-1221.

Denkova, D., Verellen, N., Silhanek, A.V. Dorpe, V., Moshchalkov, V.V. (2013). Mapping magnetic near-field distribution of plasmonic nanoantennas. ACS Nano, 7 (4): 3168-76.

Fievet, F., Langier, J., Blim, B., Beaudoin, B., Figlarz. (1989). Homogeneous and heterogeneous nucleations in the polyol process for the preparation of micron and submicron size metal particles. Solid State Ionics. 32: 198-205.

González, E., Arbiol, J. Puntes, V. (2011). Carving at the nanoscale: Sequential galvanic exchange and Kirkendall growth at room temperature. Science. 334: 1377-1380.

González, E., Merkoci, F., Arenal, R., Arbiol, J., Esteve, J., Bastús, N.G., Puntes, V. (2016). Enhanced reactivity of high-index surface platinum hollow nanoscrystals. J. Mater. Chem. A. 4: 200-208.

Ibáñez, M. \& Cabot, A. (2013). All change for nanocrystals. Science. 340: 935-936.

Liu, K., Bai, Y., Zhang, L., Yang, Z., Fan, Q., Zheng, H., Yin, Y., Gao, Ch. (2016). Porous Au-Ag nanospheres with high-density and highly accessible hotspots for SERS analysis. Nano Lett. 16 (6): 3675-3681.

Mahmoud, A., O’Neil, D., El-Sayed, M.A. (2014) Hollow and solid metallic nanoparticles in sensing and in nanocatalysis. Chem. Mater. 26 (1): 44-58.

Oh, M., Yu, T., Yu, S-H., Lim, B., Ko, K-T., Willinger, M-G., Seo, D-H., Kim, B., Cho, M., Park, J-H., Kang, K., Sung, Y-E., Pinna, N, Hyeon, T. (2013). Galvanic replacement reactions in metal oxide nanocrystals. Science. 340: 964-968.

Parak, J. W. (2011). Complex colloidal assembly. Science. 334: 1359-1360.

Wang, Y., Price A., Caruso, F. (2009). Nanoporous colloids: Building blocks for a new generation of structured materials. J. Mater. Chem., 19: 6451-6464.

Subramanian, V., Cheng, K., Lancelot, Ch., Heyte, S., Paul, S., Moldovan, S., Ersen, O., Marinova, M., Ordomsky, V., Khodakov, A. (2016). Nanoreactors: An efficient tool to control the chain-length distribution in Fischer-Tropsch synthesis. ACS Catalysis. 6 (3): 1785-1792. 
Skrabalak, S.E., Au, L. Li, X., Xia, Y. (2007) Facile synthesis of Ag nanocubes and $\mathrm{Au}$ nanocages. Nature Protocols. 2: 2182-2190.

Skrabalak, S.E., Wiley, B., Kim, M., Forno, E., Xia, Y. (2008). On the polyol synthesis of silver nanostructures: Glycolaldehyde as a reducing agent. Nano Lett. 8: 20772081.

Sperling, R.A. \& Parak, W.J. (2011). Surface modification, functionalization and bioconjugation of colloidal inorganic nanoparticles. Phil. Trans. R. Soc. A. 368: 1333-1383.
Yin., Y., Rioux, R.M., Erdonmez, C.K., Hughes, S., Somarjai, G.A., Alivisatos, A.P. (2004). Formation of hollow nanocrystals through the nanoscale Kirkendall effect. Science. 304: 711-714.

Yu, W., Yang, X., Liu, Y., Mitra, R. (2009). Electromagnetic simulation techniques based on the FDTD method. New Jersey: John Wiley \& Sons, Inc. 